\title{
UNIFYING MULTIPLIER THEOREMS OF HÖRMANDER, MARCINKIEWICZ, AND MICHLIN TYPE ${ }^{1}$
}

\author{
BY WILLIAM C. CONNETT AND ALAN L. SCHWARTZ
}

Communicated by Alberto Calderón, November 11, 1974

The study of multiplier theorems for Fourier expansions led quite naturally to the study of multiplier theorems for other orthogonal expansionsin particular the ultraspherical. In this setting several results were proved [1] , [3], [5] -but each result was the son of a Fourier theorem. Was there an "ideal" theorem that contained these? In the ultraspherical setting-there is. In order to find this "ideal" theorem two questions had to be answered: (a) what were the best possible boundary results, and (b) what method of interpolation would give intermediate results between these boundary results?

The answer to question (a) involves an extension of the Littlewood-Paley methods to this setting (Theorem $\mathbf{A}$ ) and then the establishment of the best possible result for $q=2$ (Theorem B).

The answer to question (b) involves: First, the definition of a continuous scale of Banach spaces that contain all of the standard multiplier sequences as special cases: Second, the proof that any such sequence is the restriction to the integers of a function in a local Sobolev space (Theorem C): Third, a method of interpolation for such spaces, and the proof of local stability for these spaces (Theorem D). Finally, various special calculations and interpolation theory yield multiplier theorems for parameters $(\alpha, 1 / q, 1 / p)$ lying in a convex region that includes theorems of Marcinkiewicz type, Hömander type, and Michlin type as special examples (Theorem E).

From the perspective of Fourier analysis some of these results are quite provocative. For example, Theorem E suggests Hörmander's multiplier theorem with the number of differences $=n / 2$. Another example is Theorem $\mathrm{D}$ which suggests a method of unifying the two types of multiplier theorems in Stein [4, Chapter IV].

Define the following Littlewood-Paley functions:

AMS (MOS) subject classifications (1970). Primary 42A18, 33A45; Secondary $42 \mathrm{A56}$

1 The proofs of these theorems will appear as a future Memoir of the A.M.S. 


$$
\begin{aligned}
& g_{\alpha}(f, \theta)=\left(\int_{0}^{1}\left|d_{\alpha} f(r, \theta)\right|^{2}|\log r|^{2 \alpha-1} \mid \frac{d r}{r}\right)^{1 / 2}, \\
& S(f, \theta)=\left(\int_{0}^{1}\left|d_{1} f(r, \circ)\right|^{2} * \chi_{1-r}(\theta)(1-r)^{-2 \lambda} \mid d r\right)^{1 / 2}, \\
& g_{\eta}^{*}(f, \theta)=\left(\int_{0}^{1}\left|d_{1} f(r, \circ)\right|^{2} * k_{\eta, r}(\theta)(1-r)^{-2 \lambda} \mid d r\right)^{1 / 2},
\end{aligned}
$$

where the convolution *, the measure $d \mu_{\lambda}$, the norm $\|\cdot\|_{p}$, and the Abel means $f(r, \theta)$ are those associated with ultraspherical series (see [3]), where $d_{\alpha}$ is a fractional differential operator, $\chi_{\epsilon}(\theta)=1(=0)$ if $\theta \leqslant \epsilon(>\epsilon)$, and $k_{n, r}(\theta)=\{(1-r) /(1-r+\theta)\}^{n(2 \lambda+1)}$.

Theorem A. Let $f \in L^{2} \cap L^{p}$ and $\int f d \mu_{\lambda}=0$, then

$$
\begin{aligned}
\left\|g_{\eta}^{*}(f)\right\|_{p} & \leqslant A_{p}\|f\|_{p}, \quad 2 \leqslant p<\infty, \eta>1, \\
g_{\alpha}(f, \theta) & \leqslant A_{\alpha, \beta} g_{\beta}(f, \theta), \quad \beta>\alpha, \\
S(f, \theta) & \leqslant A_{\eta} g_{\eta}^{*}(f, \theta) .
\end{aligned}
$$

We need the following spaces of sequences and functions.

Define $\left\{m_{n}\right\} \in m(q, k)$ if

$$
\begin{gathered}
\sup _{n}\left|m_{n}\right| \leqslant C_{1}, \\
\sup _{M}(1 / M) \Sigma_{M}^{2 M}\left|n^{\kappa} \Delta^{\kappa} m_{n}\right|^{q} \leqslant C_{2}^{q} .
\end{gathered}
$$

Let $\varphi(x)$ be a $C_{c}^{\infty}$ function which is used to cut off the support of other functions in a smooth way. Let $\varphi_{k}(x)=\varphi(x-k)$ be a translate of $\varphi(x)$. C.

Define $m(x) \in S(q, \alpha)$, a local Sobolev space, if $\sup _{k}\left\|\varphi_{k}(x) m\left(2^{x}\right)\right\|_{L_{\alpha}^{q}}<$

(We assume $L_{\alpha}^{q}$ is the Sobolev space when $k$ is an integer, the Bessei potential space elsewhere. See Stein [4] for notation.)

Define $\left\{m_{n}\right\} \in s(q, \alpha)$ if there exists an $m \in S(q, \alpha)$ such that $m(n)=$ $m_{n}$.

The spaces $s(q, \alpha)$ are the scale of Banach spaces that contain the multipliers of Marcinkiewicz, etc. as special cases.

THEOREM B. If $\alpha>\lambda+1 / 2$ and $\left\{m_{n}\right\} \in s(2, \alpha) f \sim \Sigma c_{n} P_{n}^{\lambda}$ and Tf $\sim$ $\Sigma m_{n} c_{n} P_{n}^{\lambda}$, then $\|T f\|_{p} \leqslant C_{p}\|f\|_{p}$ for all $1<p<\infty$.

THEOREM C. $m(q, k) \equiv s(q, k)$. 
If $[,]_{s}$ denotes the complex method of interpolation of Calderón [2], then a substitute is needed for the idea of stability, which would require that $\left[S\left(q_{0}, \alpha_{0}\right), S\left(q_{1}, \alpha_{1}\right)\right]_{s}=S(q, \alpha)$, since this may fail for these nonreflexive spaces. Thus we have the following

\section{THEOREM D. Local stability.}

$$
S(q, \alpha) \cap C_{c} \subset\left[S\left(q_{0}, \alpha_{0}\right), S\left(q_{1}, \alpha_{1}\right)\right]_{s} \subset S(q, \alpha) .
$$

Define $E_{S}$ to be the subset of $(\alpha, 1 / q, 1 / p)$ space for which $S(q, \alpha)$ defines a strong type $p$ - $p$ multiplier by restriction.

THEOREM E. The set $E_{S}$ contains the interior of the convex hull of the following endpoint or boundary results:

(a) $\alpha q=1, \alpha>0,2 \leqslant q \leqslant \infty$;

(b) $2 \leqslant q \leqslant \infty, \alpha>\lambda+1 / 2,1<p<\infty$;

(c) $q=1, \alpha=[\lambda+1]+1,1<p<\infty$;

(d) $q=1, \alpha=1,|1 / p-1 / 2|<1 / 2(2 \lambda+1)$.

That the results are best possible for $\alpha q \geqslant 2 \lambda+1$ can be given by a specific construction.

REMARK 1. In order to show that the convex region gives the best possible result for all $(\alpha, 1 / q, 1 / p)$, an improvement must be made in the theorem of Bonami and Clerc [1]. We conjecture that the following multipliers of Marcinkiewicz type is true: If $\left\{m_{n}\right\} \in s(1, \alpha)$, then $\|T f\|_{p} \leqslant C\|f\|_{p}$ for all $1<p<\infty$ if $\alpha>\lambda+1 / 2$.

REMARK 2. These results have been stated in terms of Sobolev spaces, but there is an analogous theory for other smoothness classes (for example $\left.\Lambda_{\alpha}^{p, p}\right)$

\section{REFERENCES}

1. A. Bonami and J. L. Clerc, Sommes de Cesaro et multiplicateurs, Trans. Amer. Math. Soc. 183 (1973), 223-264. $113-190$.

2. A. P. Calderón, Intermediate spaces and interpolation, Studia Math. 24 (1964),

3. W. Connett and A. Schwartz, A multiplier theorem for ultraspherical series, Studia Math. 51 (1973), $51-70$. ton, 1970.

4. E. Stein, Singular integrals and differentiability properties of functions, Prince-

5. R. Strichartz, Multipliers for spherical harmonic expansions, Trans. Amer. Math. Soc. 167 (1972), 115-124.

DEPARTMENT OF MATHEMATICS, UNIVERSITY OF MISSOURI-ST. LOUIS, ST. LOUIS, MISSOURI 63121 